\title{
FIBERED PRODUCTS OF HOMOGENEOUS CONTINUA
}

\author{
KAREN VILLARREAL
}

\begin{abstract}
In this paper, we construct homogeneous continua by using a fibered product of a homogeneous continuum $X$ with itself. The space $X$ must have a continuous decomposition into continua, and it must possess a certain type of homogeneity property with respect to this decomposition. It is known that the points of any one-dimensional, homogeneous continuum can be "blown up" into pseudo-arcs to form a new continuum with a continuous decomposition into pseudo-arcs. We will show that these continua can be used in the above construction. Finally, we will show that the continuum constructed by using the pseudo-arcs, the circle of pseudo-arcs, or the solenoid of pseudo-arcs is not homeomorphic to any known homogeneous continuum.
\end{abstract}

\section{INTRODUCTION}

A continuum is a compact, connected metric space. A continuum $X$ is homogeneous if, for any pair of points $x, y$ in $X$, there exists a homeomorphism $h:(X, x) \rightarrow(X, y)$. In this paper, we will build new homogeneous continua by using fibered products of other homogeneous continua. If $f: X \rightarrow Z$ and $g: Y \rightarrow Z$ are maps, then the fibered product of $X$ and $Y$ with respect to $f$ and $g$ is the space $\{(x, y) \in X \times Y: f(x)=g(y)\}$.

One of the new homogeneous continua is the fibered product of two pseudoarcs. A pseudo-arc is a chainable, hereditarily indecomposable continuum. A chainable continuum is a continuum which is homeomorphic to an inverse limit of arcs, and an indecomposable continuum is a continuum which is not the union of two of its proper subcontinua. A continuum is hereditarily indecomposable if every subcontinuum is indecomposable. Clearly, every nondegenerate subcontinuum of a pseudo-arc is a pseudo-arc. The pseudo-arc was first constructed by Knaster [7] in 1922. It was shown to be homogeneous by Bing [3] in 1948. Bing [2] also proved that all pseudo-arcs are homeomorphic.

A continuous decomposition of a continuum is a partition of the continuum into subcontinua such that the quotient map of the partition is both open and closed. A circle of pseudo-arcs is a continuum with a continuous decomposition into pseudo-arcs, such that the quotient space is a circle. Bing and Jones [4] constructed a circle of pseudo-arcs in 1954, and showed that any two circles of pseudo-arcs are homeomorphic, and that the circle of pseudo-arcs is homogeneous.

Received by the editors May 23, 1991.

1991 Mathematics Subject Classification. Primary 54F20.

Key words and phrases. Continuum, homogeneous, fibered product, aposyndetic continuum, continuous decomposition, pseudo-arc, circle of pseudo-arcs, solenoid of pseudo-arcs. 
A solenoid is an inverse limit of circles with covering maps as the bonding maps. In 1977, for each solenoid $S$, Rogers [11] constructed a solenoid of pseudo-arcs, that is, a homogeneous continuum with a continuous decomposition into pseudo-arcs, such that the quotient space is $S$.

In 1984, Lewis [8] generalized the above results by showing that points of any homogeneous one-dimensional continuum $X$ can be "blown up" into pseudoarcs, so that the resulting continuum is a homogeneous continuum with a continuous decomposition into pseudo-arcs such that the quotient space is $X$.

In this paper, we will show that if $Y$ is any of the continua constructed in Lewis' paper, a homogeneous continuum can be constructed as a fibered product of $Y$ with itself. In particular, we will show that the continua constructed from the pseudo-arc, the circle of pseudo-arcs, and a solenoid of pseudo-arcs are not homeomorphic to any known homogeneous continua.

Let $X$ be a continuum which has a continuous decomposition into continua, and let $f: X \rightarrow Q$ be the quotient map where $Q$ is a homogeneous continuum. We will call the following property of $X$, with respect to $f$, Property $\mathrm{H}$ :

If $h$ is any homeomorphism of $Q$, and if $h(f(x))=f(y)$, then there is a homeomorphism $\hat{h}:(X, x) \rightarrow(X, y)$ such that $f \circ \hat{h}=h \circ f$.

In this section, we will prove the following theorem.

Theorem 1. Let $X$ be a continuum with a continuous decomposition into nondegenerate continua, and let $f: X \rightarrow Q$ be the quotient map, where $Q$ is a homogeneous continuum and $X$ has Property $\mathrm{H}$ with respect to $f$. Let $\widetilde{X}=\{(x, y) \in X \times X: f(x)=f(y)\}=\bigcup\left\{f^{-1}(q) \times f^{-1}(q): q \in Q\right\}$. Then $\widetilde{X}$ is an aposyndetic, homogeneous continuum such that for each $q \in Q$,

$$
\operatorname{dim}\left(f^{-1}(q) \times f^{-1}(q)\right) \leq \operatorname{dim} \tilde{X} \leq \operatorname{dim}(X \times X) .
$$

Proof. Let $\pi_{1}: \widetilde{X} \rightarrow X$ and $\pi_{2}: \widetilde{X} \rightarrow X$ be projections onto the first and second coordinates, respectively. The map $\pi_{1}$ is open, because, if $U_{1}$ and $U_{2}$ are open subsets of $X$, then $\pi_{1}\left(\left(U_{1} \times U_{2}\right) \cap \tilde{X}\right)=U_{1} \cap f^{-1}\left(f\left(U_{2}\right)\right)$ which is an open set since $f$ is open. Also, note that for each $x \in X, \pi_{1}^{-1}(x)=\{x\} \times f^{-1}(f(x))$ is homeomorphic to a decomposition element of $X$.

The space $\widetilde{X}$ is compact, since it is a closed subset of $X \times X$. The space $\tilde{X}$ is also connected. For, if $\widetilde{X}=U \cup V$, where $U$ and $V$ are nonempty, disjoint open sets, then $\pi_{1}(U)$ and $\pi_{1}(V)$ are nonempty open sets such that $\pi_{1}(U) \cup \pi_{1}(V)=X$. If $x \in \pi_{1}(U) \cap \pi_{1}(V)$, then $\{x\} \times f^{-1}(f(x))$ intersects both $U$ and $V$. But $\{x\} \times f^{-1}(f(x))$ is connected, so it must be contained in one of these sets. Then $\widetilde{X}$ must be connected, and hence a continuum.

The space $\widetilde{X}$ is also homogeneous. If $\left(x_{1}, y_{1}\right)$ and $\left(x_{2}, y_{2}\right)$ are points of $\tilde{X}$, let $p_{1}=f\left(x_{1}\right)=f\left(y_{1}\right)$ and $p_{2}=f\left(x_{2}\right)=f\left(y_{2}\right)$. Choose a homeomorphism $h:\left(Q, p_{1}\right) \rightarrow\left(Q, p_{2}\right)$. Then, by Property $\mathrm{H}$, there are homeomorphisms $h_{x}:\left(X, x_{1}\right) \rightarrow\left(X, x_{2}\right)$ and $h_{y}:\left(X, y_{1}\right) \rightarrow\left(X, y_{2}\right)$, with $f \circ h_{x}=h \circ f=f \circ h_{y}$. We have $\left(h_{x} \times h_{y}\right)(\widetilde{X}) \subset \widetilde{X}$, for if $(x, y) \in \widetilde{X}$, then $f(x)=f(y)$, so

$$
f\left(h_{x}(x)\right)=h(f(x))=h(f(y))=f\left(h_{y}(y)\right) .
$$


Also, if $(u, v) \in \tilde{X}$, then $f(u)=f(v)$, so

$$
\begin{aligned}
h\left(f\left(h_{x}^{-1}(u)\right)\right) & =f\left(h_{x}\left(h_{x}^{-1}(u)\right)\right)=f(u)=f(v) \\
& =f\left(h_{y}\left(h_{y}^{-1}(v)\right)\right)=h\left(f\left(h_{y}^{-1}(v)\right)\right) .
\end{aligned}
$$

Since $h$ is a homeomorphism, $f\left(h_{x}^{-1}(u)\right)=f\left(h_{y}^{-1}(v)\right)$, and $\left(h_{x}^{-1}(u), h_{y}^{-1}(v)\right) \in$ $\tilde{X}$. Then $\left(h_{x} \times h_{y}\right)(\tilde{X})=\tilde{X}$, and therefore, $h_{x} \times h_{y}$ is a homeomorphism of $\tilde{X}$ that takes $\left(x_{1}, y_{1}\right)$ to $\left(x_{2}, y_{2}\right)$.

A continuum $Y$ is aposyndetic if for each pair of distinct points $x$ and $y$ in $Y$, there is a subcontinuum $S$ of $Y$ such that $x \in \operatorname{int}(S)$ and $y \in Y-S$. Jones [6] was the first to use this concept to study homogeneous continua.

We will next show that $\widetilde{X}$ must be aposyndetic. Let $(x, y)$ and $\left(x^{\prime}, y^{\prime}\right)$ be distinct points in $\tilde{X}$. Without loss of generality, assume $x \neq x^{\prime}$. Let $U$ be an open subset of $X$ such that $x \in U$ and $x^{\prime} \notin \bar{U}$. Then $\pi_{1}^{-1}(\bar{U})$ is a closed set containing $(x, y)$ in its interior, but not containing $\left(x^{\prime}, y^{\prime}\right)$. Since $\pi_{1}^{-1}(\bar{U})=\bigcup\left\{\{z\} \times f^{-1}(f(z)): z \in \bar{U}\right\}$, it is a union of connected sets. Let $\hat{h}$ be a homeomorphism of $X$ which fixes the decomposition elements, such that $\hat{h}\left(x^{\prime}\right) \neq y^{\prime}$. We can obtain $\hat{h}$ by using Property $\mathrm{H}$, and letting the function $h$ in the definition of this property be the identity homeomorphism. Then the set $A=\{(z, \hat{h}(z)): z \in X\} \subseteq \widetilde{X}$ is a continuum, since it is homeomorphic to $X$. Also, $\left(x^{\prime}, y^{\prime}\right) \notin A$, and for every $z \in \bar{U}$,

$$
A \cap\left[\{z\} \times f^{-1}(f(z))\right] \neq \varnothing .
$$

Then $\pi_{1}^{-1}(\bar{U}) \cup A$ is a continuum containing $(x, y)$ in its interior, but not containing $\left(x^{\prime}, y^{\prime}\right)$.

We also know that for each $q \in Q$, we have

$$
\operatorname{dim}\left(f^{-1}(q) \times f^{-1}(q)\right) \leq \operatorname{dim}(\tilde{X}) \leq \operatorname{dim}(X \times X),
$$

since $f^{-1}(q) \times f^{-1}(q) \subset \tilde{X} \subset X \times X$.

We can generalize the above result. If $\mathscr{A}$ is any indexing set, and $X$ is a continuum with a continuous decomposition into nondegenerate subcontinua, with quotient space the homogeneous space $Q$, and having Property $\mathrm{H}$ with respect to the quotient map $f$, then the space $\bigcup_{q \in Q} \prod_{\alpha \in \mathscr{A}} f^{-1}(q)$ is an aposyndetic, homogeneous continuum. The proof is analogous to the proof of Theorem 1. Also, suppose $X$ and $Y$ are two continua with continuous decompositions into nondegenerate subcontinua, both with quotient space the homogeneous space $Q$. Suppose $X$ has Property $\mathrm{H}$ with respect to the quotient map $f$, and $Y$ has Property $\mathrm{H}$ with respect to the quotient map $g$. Then a proof analogous to the proof of Theorem 1 shows that $\bigcup_{q \in Q}\left(f^{-1}(q) \times g^{-1}(q)\right)$ is a homogeneous continuum. However, the author is not able to conclude that this space is aposyndetic.

\section{II}

Lewis [8] has shown that if $Q$ is a one-dimensional, homogeneous continuum, then there is a one-dimensional continuum $X$ with a continuous decomposition into pseudo-arcs with quotient map $f: X \rightarrow Q$, such that $X$ satisfies Property $\mathbf{H}$ with respect to $f$. Then $\widetilde{X}$, as constructed in $\S \mathbf{I}$, would be a twodimensional, homogeneous, aposyndetic continuum. Since the pseudo-arc is 
acyclic, the Vietoris-Begle Theorem tells us that the Čech cohomology of $\tilde{X}$ is the same as the Čech cohomology of $Q$.

Three continua with which we could replace $Q$ in the above paragraph are the circle, a solenoid, and the pseudo-arc. The corresponding continua with continuous decompositions into pseudo-arcs are the circle of pseudo-arcs $(C P)$, a solenoid of pseudo-arcs $(S P)$, and the pseudo-arc of pseudo-arcs $(P)$, which is known to be homeomorphic to the pseudo-arc [9]. We will show that $\widetilde{C P}, \widetilde{S P}$, and $\widetilde{P}$ are not homeomorphic to any previously known homogeneous continuum. J. T. Rogers has informed the author that, in the case of $\widetilde{C P}$, this solves a problem posed by the late Andrew Conner about 10 years ago.

Rogers [10] has classified homogeneous continua as being locally connected, aposyndetic but not locally connected, decomposable but not aposyndetic, or indecomposable (with further subcategories for indecomposable continua.) $\mathrm{He}$ lists all the known homogeneous continua in each category. $\widetilde{C P}, \widetilde{S P}$, and $\widetilde{P}$ are two-dimensional continua and belong to the category aposyndetic, but not locally connected. They are not locally connected because their images under $\pi_{1}$ are not locally connected.

The known two-dimensional homogeneous continua in this category are products of two one-dimensional homogeneous continua, at least one of which is not locally connected, and a few other continua which contain arcs. None of $\widetilde{C P}$, $\widetilde{S P}$, and $\widetilde{P}$ can contain an arc, because the image of an arc under $\pi_{1}$ would have to be locally connected. The only locally connected subcontinua of $C P$, $S P$, or $P$ are points. If the image of an arc under $\pi_{1}$ was a point, the arc would have to be contained in a pseudo-arc, which contains no arcs.

The known one-dimensional homogeneous continua are $S^{1}$, the Menger curve, the Case continua, other Cantor set bundles over the Menger curve, the solenoids, the pseudo-arc, the circle of pseudo-arcs, the Menger curve of pseudoarcs, the Case continua of pseudo-arcs, the Cantor set bundles over the Menger curve of pseudo-arcs, and the solenoids of pseudo-arcs [10]. It is easy enough to list all of the products of two one-dimensional homogeneous continua, at least one of which is not locally connected, and note which have the cohomology of $\widetilde{C P}, \widetilde{S P}$, or $\widetilde{P}$. The continua in the list with the cohomology of $\widetilde{C P}$ are $S^{1} \times P$ and $C P \times P$. The continua with the cohomology of $\widetilde{S P}$ are $S \times P$ and $S P \times P$. The only continuum in the list with the cohomology of $\widetilde{P}$ is $P \times P$. Since $S^{1} \times P$ and $S \times P$ contain arcs, the only homogeneous continua to which $\widetilde{C P}, \widetilde{S P}$, and $\widetilde{P}$ could be homeomorphic are $C P \times P, S P \times P$, and $P \times P$, respectively.

\section{III}

In this section, we will show that $\widetilde{S P}$ is not homeomorphic to $S P \times P$, and $\widetilde{P}$ is not homeomorphic to $P \times P$. A fact that we will need in this section and the next is that the decompositions of $C P, S P$, and $P$ are terminal. This means that each subcontinuum is either contained in a decomposition element, or is a union of decomposition elements. Hence, the only proper, nondegenerate subcontinua of $C P$ and $S P$ are arcs of pseudo-arcs and pseudo-arcs.

Now we will prove a couple of lemmas. 
Lemma 1. Let $X$ be $C P, S P$, or $P$, and let $K$ be a subcontinuum of $\tilde{X}$ such that $\pi_{1}(K)$ intersects more than one decomposition element. Then $\pi_{2}(K)=$ $\pi_{1}(K)$.

Proof. Let $D$ be a decomposition element which intersects $\pi_{1}(K)$, and let $(x, y) \in K \cap \pi_{1}^{-1}(D)$. Then we must have $y \in D$; so $D$ intersects $\pi_{2}(K)$. Likewise, any decomposition element which intersects $\pi_{2}(K)$ also intersects $\pi_{1}(K)$. Since $\pi_{1}(K)$ and $\pi_{2}(K)$ are subcontinua of $X$ which intersect more than one decomposition element, and since the decomposition is terminal, $\pi_{1}(K)$ and $\pi_{2}(K)$ must each be a union of the decomposition elements it intersects, so $\pi_{1}(K)=\pi_{2}(K)$.

A map $g: Y \rightarrow Z$ between continua is weakly confluent if, for each subcontinuum $S$ of $Z$, there is a component of $g^{-1}(S)$ which maps onto $S$. Any map of a continuum onto a pseudo-arc, an arc of pseudo-arcs, or a solenoid of pseudo-arcs is weakly confluent [5].

Recall that pseudo-arcs are chainable. We will use the following fact concerning chainable continua in the next lemma: If $C_{1}$ and $C_{2}$ are subcontinua of $D \times D$, where $D$ is a chainable continuum, and the projection of $C_{1}$ onto the first coordinate is $D$, and the projection of $C_{2}$ onto the second coordinate is $D$, then $C_{1} \cap C_{2} \neq \varnothing[1]$.

Lemma 2. If $K_{1}$ and $K_{2}$ are subcontinua of $\widetilde{S P}$ or $\widetilde{P}$ such that $\pi_{1}\left(K_{1}\right)$ and $\pi_{1}\left(K_{2}\right)$ are each unions of more than one decomposition element, and $\pi_{1}\left(K_{1}\right) \cap$ $\pi_{1}\left(K_{2}\right) \neq \varnothing$, then $K_{1} \cap K_{2} \neq \varnothing$.

Proof. Since $\pi_{1}\left(K_{1}\right)$ and $\pi_{1}\left(K_{2}\right)$ must each be either a pseudo-arc, an arc of pseudo-arcs, or $S P$, and since $\pi_{2}\left(K_{2}\right)=\pi_{1}\left(K_{2}\right)$, we know that the maps $\pi_{1} \mid K_{1}$ and $\pi_{2} \mid K_{2}$ are both weakly confluent. There is a decomposition element $D \subset \pi_{1}\left(K_{1}\right) \cap \pi_{1}\left(K_{2}\right)=\pi_{1}\left(K_{1}\right) \cap \pi_{2}\left(K_{2}\right)$ since $\pi_{1}\left(K_{1}\right) \cap \pi_{1}\left(K_{2}\right) \neq \varnothing$, and $\pi_{1}\left(K_{1}\right)$ and $\pi_{1}\left(K_{2}\right)$ are each unions of decomposition elements. Then there exists subcontinua $C_{1}$ of $K_{1}$ and $C_{2}$ of $K_{2}$ such that $\pi_{1}\left(C_{1}\right)=D$ and $\pi_{2}\left(C_{2}\right)=D$. Then $\pi_{2}\left(C_{1}\right) \subset D$ and $\pi_{1}\left(C_{2}\right) \subset D$, since the coordinates of points of $\widetilde{P}$ and $\widetilde{S P}$ must come from the same decomposition element. Then $C_{1}$ and $C_{2}$ are subcontinua of $D \times D$ such that $\pi_{1}\left(C_{1}\right)=D$ and $\pi_{2}\left(C_{2}\right)=D$. Since $D$ is chainable, we must have $C_{1} \cap C_{2} \neq \varnothing$. Then $K_{1} \cap K_{2} \neq \varnothing$.

Let $X$ be $S P$ or $P$. Let $p_{1}: X \times P \rightarrow X$ and $p_{2}: X \times P \rightarrow P$ be the projections. If $x \in X$, we will call $p_{1}^{-1}(x)$ a vertical slice of $X \times P$, and if $q \in P$, we will call $p_{2}^{-1}(q)$ a horizontal slice of $X \times P$.

Theorem 2. $\widetilde{S P}$ is not homeomorphic to $S P \times P$, and $\widetilde{P}$ is not homeomorphic to $P \times P$.

Proof. Let $X$ be $S P$ or $P$, and assume $\sigma: X \times P \rightarrow \tilde{X}$ is a homeomorphism.

Step 1. We will show that if $h_{1}$ and $h_{2}$ are distinct horizontal slices of $X \times P$, and both $\pi_{1}\left(\sigma\left(h_{1}\right)\right)$ and $\pi_{1}\left(\sigma\left(h_{2}\right)\right)$ are unions of more than one decomposition element, then $\pi_{1}\left(\sigma\left(h_{1}\right)\right)$ and $\pi_{1}\left(\sigma\left(h_{2}\right)\right)$ do not intersect.

If $\pi_{1}\left(\sigma\left(h_{1}\right)\right)$ and $\pi_{1}\left(\sigma\left(h_{2}\right)\right)$ intersect, then by Lemma 2, $\sigma\left(h_{1}\right)$ and $\sigma\left(h_{2}\right)$ intersect. But $h_{1}$ and $h_{2}$ are distinct horizontal slices, and $\sigma$ is a homeomorphism, so $\left(\sigma\left(h_{1}\right)\right)$ and $\left(\sigma\left(h_{2}\right)\right)$ cannot intersect.

Step 2. We will show that there is at least one horizontal slice $h$ of $X \times P$ such that $\pi_{1}(\sigma(h))$ is contained in a decompostion element. 
If for every horizontal slice $h$ of $X \times P, \pi_{1}(\sigma(h))$ is a union of more than one decomposition element, then Step 1 tells us that for each horizontal slice $h, \sigma(h)=\pi_{1}^{-1}\left(\pi_{1}(\sigma(h))\right)$. But $\sigma(h)$ must be one-dimensional, while $\pi_{1}^{-1}\left(\pi_{1}(\sigma(h))\right)$ is two-dimensional, since $\pi_{1}(\sigma(h))$ is a union of more than one decomposition element. Hence, there is a horizontal slice $h$ such that $\pi_{1}(\sigma(h))$ is contained in a decomposition element.

Step 3. Now we prove the theorem. Let $h$ be a horizontal slice of $X \times P$ such that $\pi_{1}(\sigma(h))$ is contained in a decomposition element $D$. Let $v_{1}$ be a vertical slice of $X \times P$ such that $\pi_{1}\left(\sigma\left(v_{1}\right)\right)$ intersects $X \backslash D$. Since $\pi_{1}^{-1}(X \backslash D)$ is two-dimensional, $\pi_{1}^{-1}(X \backslash D) \not \subset \sigma\left(v_{1}\right)$, so there exists a vertical slice $v_{2}$ of $X \times P$ distinct from $v_{1}$ such that $\pi_{1}\left(\sigma\left(v_{2}\right)\right)$ intersects $X \backslash D$. Both $\sigma\left(v_{1}\right)$ and $\sigma\left(v_{2}\right)$ must intersect $\sigma(h)$, since both $v_{1}$ and $v_{2}$ intersect $h$, so $\pi_{1}\left(\sigma\left(v_{1}\right)\right)$ and $\pi_{1}\left(\sigma\left(v_{2}\right)\right)$ each also intersect $D$. Since $\pi_{1}\left(\sigma\left(v_{1}\right)\right)$ and $\pi_{1}\left(\sigma\left(v_{2}\right)\right)$ each also intersect $X \backslash D$, they must each be unions of more than one decomposition element, and must each contain $D$. Then by Lemma 2, $\sigma\left(v_{1}\right) \cap \sigma\left(v_{2}\right) \neq \varnothing$. But this is a contradiction since $v_{1} \cap v_{2}=\varnothing$. Hence, there can be no homeomorphism $\sigma: X \times P \rightarrow \widetilde{X}$.

In this section, we will prove that $C P \times P$ is not homeomorphic to $\widetilde{C P}$. We will use a lemma similar to Lemma 2 . Since $C P$ does not satisfy the property that every map of a continuum onto it is weakly confluent, the hypothesis for this lemma is more restrictive than the hypothesis of Lemma 2.

Lemma 3. If $K_{1}$ and $K_{2}$ are subcontinua of $\widetilde{C P}$ such that $\pi_{1}\left(K_{1}\right)$ and $\pi_{1}\left(K_{2}\right)$ are arcs of pseudo-arcs, and $\pi_{1}\left(K_{1}\right) \cap \pi_{1}\left(K_{2}\right) \neq \varnothing$, then $K_{1} \cap K_{2} \neq \varnothing$.

Proof. The proof is analogous to the proof of Lemma 2.

If $p_{1}: C P \times P \rightarrow C P$ and $p_{2}: C P \times P \rightarrow P$ are the projections, then for each $x \in C P$, we will call $p_{1}^{-1}(x)$ a vertical slice of $C P \times P$, and for each $q \in P$, we will call $p_{2}^{-1}(q)$ a horizontal slice of $C P \times P$.

Theorem 3. $C P \times P$ is not homeomorphic to $\widetilde{C P}$.

Proof. Assume $\sigma: C P \times P \rightarrow \widetilde{C P}$ is a homeomorphism.

Step 1 . We will show that if $k$ is a horizontal or vertical slice of $C P \times P$, then $\pi_{1}(\sigma(k))$ is contained in a decomposition element.

Suppose $\pi_{1}(\sigma(k))$ is not contained in a decomposition element. Then $\pi_{1}(\sigma(k))$ contains an arc of pseudoarcs $A$. Choose a sequence $k_{n}$ of vertical slices, if $k$ is vertical, or horizontal slices, if $k$ is horizontal, of $C P \times P$, such that $k_{n} \neq k$ for all $n$, and $k_{n} \rightarrow k$. Since $f\left(\pi_{1}\left(\sigma\left(k_{n}\right)\right)\right) \rightarrow f\left(\pi_{1}(\sigma(k))\right)$, we must have, for $n$ large, $f\left(\pi_{1}\left(\sigma\left(k_{n}\right)\right)\right)$ is nondegenerate. Also, $\operatorname{since} \operatorname{int}(A)$ is an open set containing points of the limit set of $\left\{\pi_{1}\left(\sigma\left(k_{n}\right)\right)\right\}$, for $n$ large, $\pi_{1}\left(\sigma\left(k_{n}\right)\right)$ must intersect $\operatorname{int}(A)$. Choose $n$ so that $\pi_{1}\left(\sigma\left(k_{n}\right)\right)$ contains an arc of pseudoarcs intersecting $\operatorname{int}(A)$, and choose a decompostion element $D \subset \operatorname{int}(A) \cap \pi_{1}\left(\sigma\left(k_{n}\right)\right)$. Let $S$ be a component of $\pi_{1}^{-1}(A) \cap \sigma(k)$, and $S_{n}$ be a component of $\pi_{1}^{-1}(A) \cap \sigma\left(k_{n}\right)$, such that $\pi_{1}(S)$ and $\pi_{1}\left(S_{n}\right)$ each intersect $D$. We will show that $\pi_{1}(S)$ and $\pi_{1}\left(S_{n}\right)$ must each be arcs of pseudo-arcs containing $D$. 
If $\pi_{1}(S)$ is not an arc of pseudo-arcs, then $\pi_{1}(S) \subset D$, and hence $S \neq \sigma(k)$. If $Y$ is a subcontinuum of $\sigma(k)$ properly containing $S$, then $\pi_{1}(Y)$ must intersect the complement of $A$, since $S$ is a component of $\pi_{1}^{-1}(A) \cap \sigma(k)$. Since $S$ is a proper subcontinuum of $\sigma(k)$, a space homeomorphic to either a pseudo-arc or a circle of pseudo-arcs, we can find a decreasing sequence $\left\{Y_{n}\right\}$ of subcontinua of $\sigma(k)$, each properly containing $S$, such that $Y_{n} \rightarrow S$. Then $\pi_{1}\left(Y_{n}\right) \rightarrow \pi_{1}(S)$. However, for each $n, \pi_{1}\left(Y_{n}\right)$ intersects the complement of $A$, while $\pi_{1}(S) \subset D \subset \operatorname{int}(A)$. Since this is impossible, $\pi_{1}(S)$ must be an arc of pseudo-arcs. Likewise, we can show that $\pi_{1}\left(S_{n}\right)$ is an arc of pseudo-arcs.

Since both $\pi_{1}(S)$ and $\pi_{1}\left(S_{n}\right)$ are arcs of pseudo-arcs containing $D$, by Lemma 3, $S \cap S_{n} \neq \varnothing$, and $\sigma(k) \cap \sigma\left(k_{n}\right) \neq \varnothing$. But $k$ and $k_{n}$ are distinct, so this is impossible. Therefore, $\sigma(k)$ must be contained in a decomposition element.

Step 2. Now we prove the theorem. Let $h$ be a horizontal slice of $C P \times$ $P$. Then $\pi_{1}(\sigma(h))$ is contained in a decomposition element $D$. If $v$ is any vertical slice of $C P \times P, \pi_{1}(\sigma(v))$ must intersect $D$ since $v$ intersects $h$. Since $\pi_{1}(\sigma(v))$ is contained in a decomposition element, $\pi_{1}(\sigma(v)) \subset D$. Since $C P \times P$ is a union of vertical slices, we have $\sigma(C P \times P) \subset \pi_{1}^{-1}(D)$, so $\sigma$ could not be a homeomorphism.

Thus, $\widetilde{C P}, \widetilde{S P}$, and $\widetilde{P}$ are new examples of homogeneous continua.

\section{ACKNOWLEDGMENT}

It is acknowledged that this research was a dissertation done at Tulane University under the direction of Professor James T. Rogers, Jr.

\section{REFERENCES}

1. D. P. Bellamy and J. M. Lysko, Factorwise rigidity of the product of two pseudo-arcs, Topology Proc. 8 (1983), 21-27.

2. R. H. Bing, Concerning hereditarily indecomposable continua, Pacific J. Math. 1 (1951), 43-51.

3. ___ A homogeneous indecomposable plane continuum, Duke Math. J. 15 (1948), 729-742.

4. R. H. Bing and F. B. Jones, Another homogeneous plane continuum, Trans. Amer. Math. Soc. 90 (1959), 171-192.

5. J. Grispolakis and E. D. Tymchatyn, On confluent mappings and essential mappings-a survey, Rocky Mountain J. Math. 11 (1981), 131-153.

6. F. B. Jones, Homogeneous plane continua, Proc. Auburn Topology Conf., Auburn Univ., 1969.

7. B. Knaster, Un continua dont tout sous-continu est indecomposable, Fund. Math. 3 (1922), 247.

8. W. Lewis, Continuous curves of pseudo-arcs, Houston J. Math. 11 (1985), 91-99.

9. - The pseudo-arc of pseudo-arcs is unique, Houston J. Math. 10 (1984), 227-233.

10. J. T. Rogers, Homogeneous continua, Topology Proc. 8 (1983), 213-233.

11. __ Solenoids of pseudo-arcs, Houston J. Math. 3 (1977), 531-537.

Department of Mathematics and Computer Science, Loyola University, New Orleans, LOUISIANA 70118

E-mail address: villarre@loyno.edu 